\title{
Analysis of Cumulative Fluid Balance Impact on the Stability of Gastrointestinal Tract Anastomosis
}

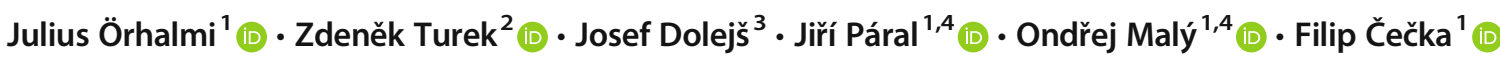

Received: 17 September 2020 / Accepted: 22 March 2021 / Published online: 30 March 2021

(C) Association of Surgeons of India 2021

\begin{abstract}
Anastomotic leakage is a serious postoperative complication following a low anterior resection of rectum. Current research in colorectal surgery focuses on searching for techniques which could minimize the risk of leakage. The main objective of this study was to evaluate the impact of cumulative fluid balance on microcirculatory changes at the anastomotic site.This experimental study used 15 domestic pigs; all of the animals underwent rectal resection. Then the animals were divided into three groups and received IV crystalloids at various rates, i.e. $5 \mathrm{ml} / \mathrm{kg} / \mathrm{h} ; 10 \mathrm{ml} / \mathrm{kg} / \mathrm{h}$; and $20 \mathrm{ml} / \mathrm{kg} / \mathrm{h}$. Large bowel micro-perfusion was measured using laser Doppler flowmetry during and following the surgical procedure. The experiments were successfully performed in all 15 cases. No animal died during the procedure or during the follow-up. We found no differences in micro-perfusion of the bowel between the experimental groups during the surgical procedure and during the follow-up period after the surgery. A significant decrease in micro-perfusion was observed after the transection of the inferior mesenteric artery in all the groups; again, no differences between the groups were observed.We did not confirm the original hypothesis that excessive load of IV crystalloids during the surgery would have a negative effect on bowel micro-perfusion and thus a negative effect on the healing of the anastomosis. However, laser Doppler flowmetry was found to have high accuracy in measuring tissue microcirculation and has the potential to be used in clinical practice.
\end{abstract}

Keywords Laser Doppler flowmetry $\cdot$ Anastomotic leakage $\cdot$ Blood flow $\cdot$ Low anterior resection

\section{Introduction}

The incidence of anastomotic leakage (AL) following rectal resection is documented to occur in $11 \%$ of patients [1], the highest incidences of which occur in the distal and middle third of the rectum. The primary etiological factor for development of AL is a local ischemia of the bowel. Well-known risk factors for dehiscence include male gender, smoking, diabetes, obesity, malnutrition, chronic obstructive pulmonary disease, use of immunosuppressants, radiotherapy, and resection of the distal third of the rectum [2]. The impact of infusion volume on anastomotic stability during perioperative and postoperative periods has not yet been studied in detail. Anastomotic oedema and microcirculatory disorders in the

1 Department of Surgery, Faculty of Medicine and University Hospital Hradec Králové, Sokolská 581, 50005 Hradec Králové, Czech Republic

2 Department for Anaesthesiology, Intensive Care and Resuscitation, Faculty of Medicine and University Hospital Hradec Králové, Hradec Králové, Czech Republic

3 Department of Informatics and Quantitative Methods, Faculty of Informatics and Management, University of Hradec Králové, Hradec Králové, Czech Republic

4 Department of Military Surgery, Faculty of Military Health Sciences, University of Defence, Hradec Králové, Czech Republic 
anastomotic region can be expected during administration of large volumes of crystalloids or rapidly administered crystalloids [3]. Currently, laser Doppler flowmetry (LDF) is the most reliable and accurate technique available for monitoring microcirculation in the region of the large bowel or rectum [4].

The objective of this study was to verify the impact of cumulative fluid balance on microcirculatory changes at anastomotic sites in the wall of the large bowel. A further objective was to explore the potential use of LDF in human clinical practice during the perioperative and postoperative periods.

\section{Methods}

The experimental study was conducted in accordance with the Protection of Animals Against Cruelty Act No. 246/92 Coll., as amended. The study was approved by the joint departmental committee of the Faculty of Military Health Sciences and the Charles University Faculty of Medicine in Hradec Králové, Czech Republic. The study model included 15 female domestic pigs (Sus scrofa f. domestica) weighing 31-42 $\mathrm{kg}$ each. All animals received human care according to the criteria outlined in the "Guide for the Care and Use of Laboratory Animals" prepared by the National Academy of Sciences. Animals' health certificates were received. The animals were completely healthy, with no signs of infectious disease, and they were examined by a vet prior to inclusion in the study. The animals were kept under standard conditions. Before the experiment, the animals were first acclimated to reduce the influence of transport and handling stress on the results of the study.

The animals were fasted $24 \mathrm{~h}$ prior to the experiment with unlimited access to water. General anaesthesia was induced using an intramuscular administration of $20 \mathrm{mg} / \mathrm{kg}$ of ketamine (Narkamon, Zentiva, Prague, Czech Republic), 4 $\mathrm{mg} / \mathrm{kg}$ of azaperone (Stresnil, Janssen Pharmaceutica, Beerse, Belgium), and $0.05 \mathrm{mg} / \mathrm{kg}$ of atropine (Atropin, Hoechst-Biotika, Martin, Slovakia), followed by a cannula insertion into the peripheral vein to ensure venous access. The pigs were orotracheally intubated with a Müller long blade laryngoscope, and the lungs were ventilated with a Servo 900C ventilator (Siemens-Elema AB, Solna, Sweden) at a tidal volume of $10-15 \mathrm{ml} / \mathrm{kg}$ and with an inspired oxygen fraction $\left(\mathrm{FiO}_{2}\right)$ of $40 \%$.

During the surgical part of the experiment, anaesthesia and analgesia were maintained with continuous intravenous (IV) infusions of midazolam at $0.3 \mathrm{mg} / \mathrm{kg} / \mathrm{h}$ and fentanyl at 30 $\mu \mathrm{g} / \mathrm{kg} / \mathrm{h}$. Postoperatively, the dosage of fentanyl was reduced to $5 \mu \mathrm{g} / \mathrm{kg} / \mathrm{h}$, and a neuromuscular blockade was induced by continuous IV infusion of pancuronium bromide at $0.2 \mathrm{mg} / \mathrm{kg}$ / h. The right femoral artery was cannulated for blood sampling and invasive blood pressure monitoring. The right jugular vein was cannulated for pulmonary artery catheterization and insertion of a central venous catheter (pressure transducer, Gabarith PMSET 1DT-XX, Becton Dickenson, Singapore; Datex-Ohmeda $\mathrm{S} / 5$ monitoring system, Instrumentarium Corp, Helsinki, Finland). Electrodes were attached to measure electrocardiogram signals, oxygen saturation, and rectal body temperature. Cystostomy and urinary catheterization were performed perioperatively through a middle laparotomy.

Preoperative blood samples were taken from all pigs to determine serum lactate concentrations, $\mathrm{pH}$, base excess, and $\mathrm{PaO}_{2}$. In addition, a Swan-Ganz catheter was used to measure cardiac output. The initial step in the procedure involved irrigating the rectum with an antiseptic Betadine solution (10\% povidone-iodine, Egis Pharmaceuticals Ltd., Budapest, Hungary) and evacuating residual stool from the rectum.

The abdominal cavity was accessed through a middle laparotomy, and a laser Doppler electrode was attached to the distal region of the sigmoid colon. The electrodes were then attached to the rectal wall using 4-0 monofilament sutures (Premilene, B. Braun Melsungen AG, Melsungen, Germany) as shown in Fig. 1. The electrode was then connected to a tissue perfusion monitor (Moor Instruments Ltd, Devon, UK), and tissue micro-perfusion values at the beginning of the experiment were read and recorded (baseline value). The inferior mesenteric artery (IMA) and inferior mesenteric vein (IMV) were then ligated, and tissue micro-perfusion values were recorded every $30 \mathrm{~min}$ following the IMA and IMV ligation for a total of $120 \mathrm{~min}$. The values were then recorded every $60 \mathrm{~min}$ for the next $120 \mathrm{~min}$.

The rectum and mesorectum were mobilized en bloc down to the pelvic floor, at the level where the rectum was ligated, using a Contour®-curved stapler (Johnson \& Johnson Inc., Cornelia, GA, USA). The rectal stump was irrigated with an antiseptic Betadine solution, during which the staple line was monitored for leakage. The ligated rectum was then extracted, followed by ligation of the large bowel in the distal region of the sigmoid colon. A circular stapler cap was inserted into the ligated lumen of the large bowel, and the lumen was closed with a circular, monofilament suture.

An LDF electrode was attached to the bowel approximately $1 \mathrm{~cm}$ proximal to the expected anastomosis site. An end-toend stapled anastomosis was performed using a 25-mm circular stapler (Circular Stapler CDH25 Johnson \& Johnson, division of Ethicon, Somerville, NJ, USA). Following construction of the anastomosis, LDF values were recorded again. Afterwards, saline was instilled in the abdominal cavity, and air was introduced into the rectum to test for anastomotic air leakage and verify anastomotic integrity. After saline aspiration, the electrodes were removed, and the laparotomy was closed with a continuous suture.

The animals were divided into three groups with 5 animals each. The animals in the first group received crystalloid infusion (Infusio Hartmanni, Medicamenta, Vysoké Mýto, Czech Republic) at a constant rate of $5 \mathrm{ml} / \mathrm{kg}$ throughout the 
Fig. 1 Laser Doppler probe fixed on the large bowel wall

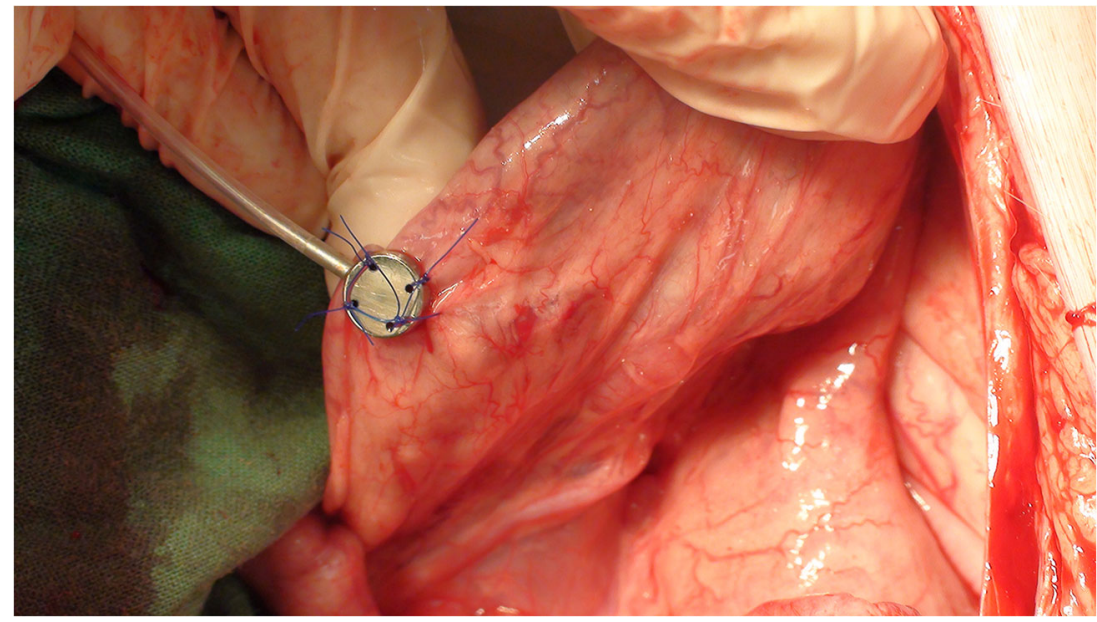

entire operation and postoperative follow-up period. The animals in the second group received the same treatment, only the infusion rate was $10 \mathrm{ml} / \mathrm{kg}$. And the animals in the third group received the same treatment, only the infusion rate was $20 \mathrm{ml} /$ $\mathrm{kg}$. At the end of the experiment, the animals were euthanized by an IV administration of T61 (Hoechst, Frankfurt-on-Main, Germany).

In all 3 groups $(5 \mathrm{ml} / \mathrm{kg} / \mathrm{h}, 10 \mathrm{ml} / \mathrm{kg} / \mathrm{h}$, and $20 \mathrm{ml} / \mathrm{kg} / \mathrm{h})$, the null hypothesis was tested; i.e., the mean values were not different between the groups. This was tested using ANOVA test which was performed separately for each of the 6 measurements; i.e., baseline, $30 \mathrm{~min}, 60 \mathrm{~min}, 120 \mathrm{~min}$, $180 \mathrm{~min}$, and $240 \mathrm{~min}$.

Besides the baseline measurement, a standard ANOVA analysis was performed using Tukey's post hoc test. The systemic hemodynamic data and fluid intake volumes are presented as mean with standard deviation (SD). Non-normally distributed microcirculatory data is presented as the mean with a $95 \%$ confidence interval (CI). Baseline and consecutive data were compared with the variance analysis (ANOVA) and the Kruskal-Wallis test for repeated measurements; an unpaired Student's $t$-test was used to analyse differences between treatment groups. $p$ values less than 0.05 were considered statistically significant. Statistical analyses were performed using SPSS Statistics 22.0 (SPSS Inc., Chicago, IL, USA).

\section{Results}

The experiment was successfully completed on all 15 animals. Throughout the course of the experiment, the animals were monitored, and their status was recorded. Oxygen saturation, cardiac events, body temperature, blood pressure, central venous pressure, pulmonary artery pressure, and diuresis were not different between the groups. All the measurements of large bowel perfusion were performed as planned, i.e. a total of 6 experimental data points, plus a baseline, for each animal.
First, the homogeneity of scatter was tested with Levene's test for homogeneity of variances. A statistically significant difference in homogeneity scatter was found only for the baseline measurement. The results are summarized in Table 1.

The results of the ANOVA test showed that the mean values were not different at baseline and at 30,180, and $240 \min (p>0.05)$. On the other hand, differences in group mean values were found at the two intermediate time points of $60 \mathrm{~min}$ and $120 \mathrm{~min}(p<0.05)$.

No differences in large bowel micro-perfusion between the groups were found. Thus, the original hypothesis that administration of large volumes of crystalloids would have a negative effect on anastomotic stability and healing during the postoperative period was not confirmed. A significant decrease in microcirculation was observed in all animals after IMA ligation as shown in Fig. 2.

\section{Discussion}

There are multiple causes of AL in large bowel and rectum anastomosis. AL contributes significantly to postoperative

Table 1 Results of one-way analysis of variance ANOVA

\begin{tabular}{lll}
\hline Time & Homogeneity of variances, $p$ value & ANOVA, $p$ value \\
\hline Baseline & $\mathbf{0 . 0 0 2}$ & 0.887 \\
$30 \mathrm{~min}$ & 0.987 & 0.156 \\
$60 \mathrm{~min}$ & 0.083 & $\mathbf{0 . 0 1 3}$ \\
$120 \mathrm{~min}$ & 0.116 & $\mathbf{0 . 0 3 6}$ \\
$180 \mathrm{~min}$ & 0.368 & 0.157 \\
$240 \mathrm{~min}$ & 0.284 & 0.708 \\
\hline
\end{tabular}

Levene's test for homogeneity of variances was performed. Significant difference was found only for the baseline measurement. ANOVA test analyzed the differences of the mean values. Statistically significant differences were found for the measurements at 60 minutes and 120 minutes 
Fig. 2 Tissue perfusion measured by laser Doppler flowmetry

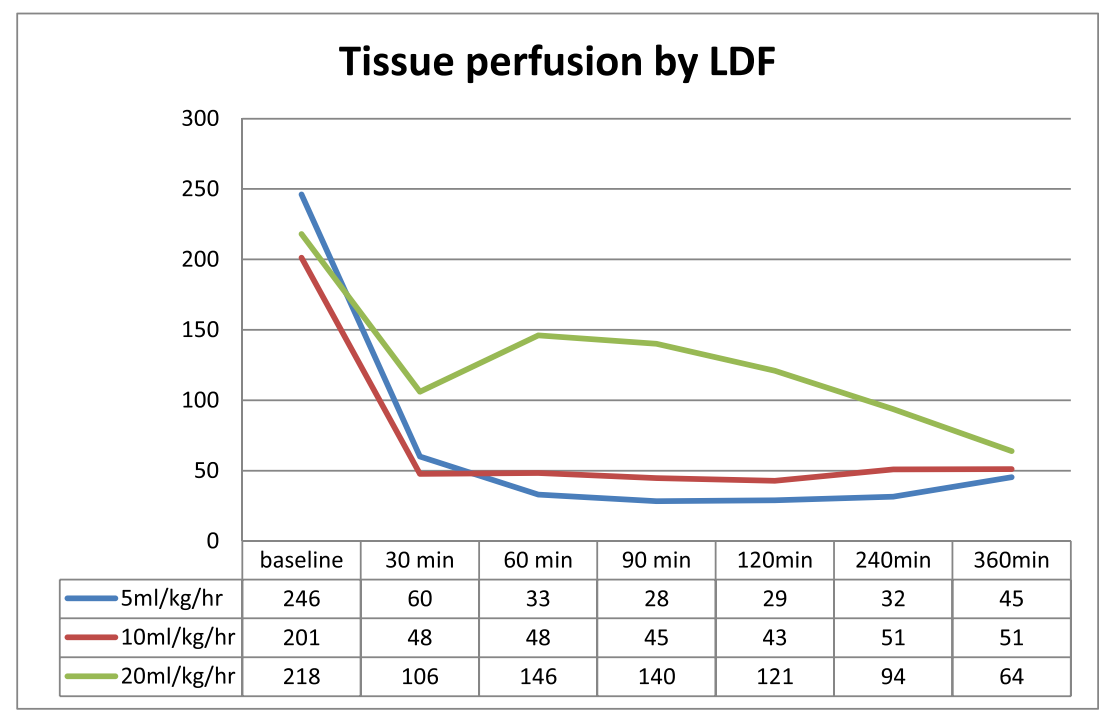

mortality and morbidity. In some patients, it even leads to functional disturbances or the need for a permanent colostomy. It is important to assess all risk factors and eliminate or minimize them. The main cause for AL is ischemia in the anastomotic region. Ischemia can develop due to capillary thrombosis in the site of anastomosis. Prolonged hypotension or hypovolemia, associated with greater blood loss, may also lead to circulatory disorders in the anastomotic region.

The impact of infusion volume and infusion rates on anastomotic stability in the perioperative and postoperative periods has not yet been sufficiently investigated [4]. Oedema and, thus, microcirculatory disorders in the anastomotic region can be expected during administration of large crystalloid volumes or rapid crystalloid infusions. There are several methods for monitoring tissue perfusion in the preoperative, perioperative, and postoperative periods that have been used experimentally, including macro-hemodynamic indicators such as pulse-induced contour cardiac output (PiCCO) and lithium dilution cardiac output (LiDCO) measurements; additionally, tissue perfusion laboratory markers can be monitored. LDF appears to be the most reliable method for monitoring the microcirculation in the large bowel or rectum. Other methods include sidestream dark field imaging, which is based on orthogonal polarization spectral imaging, and intestinal microdialysis [5-9]. Larger studies, as well as comparisons of individual methods in larger samples, have yet to be conducted.

LDF is a non-invasive diagnostic method for measuring tissue blood flow, which is based on measuring the Doppler effect that occurs when moving red blood cells cause a shift in the frequency of incident light. LDF can record the flow, speed, and concentration of moving red blood cells. These values are extracted from the power spectrum via photographic flow fluctuations that are produced when light coming from the diode illuminator is reflected onto a photodetector.
There are 2 techniques used to measure perfusion in clinical practice: laser Doppler perfusion monitoring (LDPM) and laser Doppler perfusion imaging (LDPI). LDF is able to measure real-time tissue microcirculation and perfusion changes. The drawbacks of LDF measurements are associated with a larger probe diameter and the need to fix the probe to the tissue (in our case, the large bowel) during measurements. A new type of probe can also be used for laparoscopic procedures. Another disadvantage is the lack of postoperative monitoring, since the LDF probe must be left in situ [10-13].

In everyday clinical practice, fluorescence methods using indocyanine green (ICG), such as Pinpoint (Novadaq, Mississauga, ON, Canada), intraoperative near infrared (NIR), fluorescence imaging of perfusion, or the Karl Storz NIR-ICG fluorescence imaging system (Karl Storz, Tuttlingen, Germany), are frequently encountered. The above-mentioned systems are limited in that they only evaluate final blood flow, do not monitor the dynamics of the process, and cannot be calibrated. Further potential issues include subjective image evaluation, limitations in viewing less accessible locations, or viewing an obscured anastomotic region (most often by fatty tissue). Nevertheless, in practice, their clinical application is easier [14].

A common drawback shared by all these methods, relative to anastomoses, is the absence of threshold values that can indicate if there is an adequate blood supply for timely healing of an anastomotic site, whether another solution is required (e.g. anastomotic re-resection) or when an anastomosis should be ruled out altogether.

Regarding the values recorded after IMA ligation, it must be noted that there are differences between porcine and human vascular systems. Pigs lack a developed arcade system; thus, they are prone to develop ischemia after high IMA ligation. However, it should also be noted that all pigs used in our study were young, clinically healthy specimens with no sign of 
disease in the large bowel or rectum. The surgical procedures were carried out with optimal preoperative preparation. Obviously, this differs significantly from conditions encountered with human patients, the majority of whom are of advanced age, frequently have multiple comorbidities with pathological afflictions of the walls of the large bowel or rectum, and are often being treated subsequent to radiotherapy.

\section{Conclusion}

The original hypothesis of a blood perfusion disorder developing in the large bowel anastomotic site after administration of large fluid volumes was not confirmed. Nonetheless, we did observe a significant decrease in the blood supply of the large bowel after high inferior mesenteric artery (IMA) ligation. Laser Doppler flowmetry was found to be an accurate method for measuring tissue microcirculation and has the potential to be used in clinical practice.

Funding This study was supported by the Charles University project UK PROGRES Q40/04.

\section{Declarations}

Ethics Approval The study was approved by the Institutional Animal Care and Use Committees (Committees for IAC \& U) of the University of Defence Faculty of Military Health Sciences and the Charles University Faculty of Medicine in Hradec Králové, Czech Republic. All procedures were conducted in accordance with Act No 246/1992 Coll. on the Protection of Animals Against Cruelty. Written informed consent to use the animals in this study was issued by the University of Defence Faculty of Military Health Sciences and the Charles University Faculty of Medicine in Hradec Králové, Czech Republic, which was the owner of the animals. The manuscript adheres to the ARRIVE guidelines for the reporting of animal experiments.

Conflict of Interest The authors declare no competing interests.

\section{References}

1. Meyer J, Naiken S, Christou N, Liot E, Toso C, Buchs NC et al (2019) Reducing anastomotic leak in colorectal surgery: the old dogmas and the new challenges. World J Gastroenterol 25:50175025
2. Sciuto A, Merola G, De Palma G, Sodo M, Pirozzi F, Bracale UM et al (2018) Predictive factors for anastomotic leakage after laparoscopic colorectal surgery. World J Gastroenterol 24:2247-2260

3. Marjanovic G (2009) Impact of different crystalloid volume regimes on intestinal anastomotic stability. Ann Surg 249:181-185

4. Rutegard M, Hassmen N, Hemmingsson O, Haapamaki MM, Matthiessen P, Rutegard J (2016) Anterior resection for rectal cancer and visceral blood flow: an explorative study. Scand J Surg 105: 78-83

5. Šitina M, Turek Z, Černý V, Pař́zková R (2011) In situ assessment of the brain microcirculation in mechanically ventilated rabbits using sidestream dark-field (SDF) imaging. Physiol Res 60:75-81

6. Dworkin MJ, Allen-Mersh TG (1996) Effect of inferior mesenteric artery ligation on blood flow in the marginal artery-dependent sigmoid colon. J Am Coll Surg 183:357-360

7. Boyle NH, Manifold D, Jordan MH, Mason RC (2000) Intraoperative assessment of colonic perfusion using scanning laser Doppler flowmetry during colonic resection. J Am Coll Surg 191: 504-510

8. Turek Z, Černý V, Pař́zková R (2011) Prolonged hypervolemic hemodilution decreases functional capillary density of ileal mucosa in pigs revealed by sidestream dark-field imaging. J Zhejiang UnivSci B 12:867-874

9. Post IL, Verheijen PM, Pronk A, Siccama I (2012) Intraoperative blood pressure changes as a risk factor for anastomotic leakage in colorectal surgery. Int J Colorectal Dis 27:765-772

10. Karliczek A, Benaron DA, Baas PC (2010) Intraoperative assessment of microperfusion with visible light spectroscopy for prediction of anastomotic leakage in colorectal anastomoses. Colorectal Dis 12:1018-1025

11. Seike K, Koda K, Saito N, Oda K (2007) Laser Doppler assessment of the influence of division at the root of the inferior mesenteric artery on anastomotic blood flow in rectosigmoid cancer surgery. Int J Colorectal Dis 22:689-697

12. Corbett E, Barry B, Pollard S, Lodge J (2000) Laser Doppler flowmetry is useful in the clinical management of small bowel transplantation. Gut 47:580-583

13. Tsujinaka S, Kawamura YJ, Tan KY (2012) Proximal bowel necrosis after high ligation of the inferior mesenteric artery in colorectal surgery. Scand J Surg 101:21-25

14. Allison AS, Bloor C, Faux W (2010) The angiographic anatomy of the small arteries and their collaterals in colorectal resections: some insights into anastomotic perfusion. Ann Surg 251:1092-1097

Publisher's Note Springer Nature remains neutral with regard to jurisdictional claims in published maps and institutional affiliations. 